\title{
VAGINAL ECTOPIC URETER DIAGNOSED BY MAGNETIC RESONANCE IMAGING IN A CHILD WITH COMPLETE TRANSVERSE VAGINAL
} SEPTUM AND HYDROCOLPOS

\author{
lara Regina Siqueira Lucena ${ }^{1}$, Juliana Ávila Duarte ${ }^{1}$, Eliziane Emy \\ Takamatu², Clotilde Druck Garcia ${ }^{3}$, Eduardo Correa Costa ${ }^{2}$, \\ Álvaro Porto Alegre Furtado ${ }^{1}$, Jose Carlos Soares Fraga²
}

\begin{abstract}
Ectopic ureter draining into the vagina is a rare cause of urinary incontinence. Some cases have been reported in association with malformations of the genitourinary tract, but very few published cases are associated with vaginal septum. Our article describes the case of a girl who presented with hydrocolpos shortly after birth and was found to have a complete transverse vaginal septum. Despite successful correction of this genital anomaly, the patient developed persistent dribbling of clear fluid from the genital area. Extensive testing revealed unilateral kidney dysplasia and probable genital ectopia of the corresponding ureter. Magnetic resonance imaging (MRI) showed insertion of the ureter into the upper third of the vagina. The patient underwent nephroureterectomy of the dysplastic kidney with subsequent improvement in urinary incontinence. Ureteral ectopia is difficult to diagnose with conventional imaging methods (plain radiography, ultrasound, and CT). MRI has proved to be an excellent method for assessment of genitourinary tract conditions, particularly when other diagnostic modalities have failed or are limited, due to the high resolution of MRI scans and the possibility of native image acquisition on all three orthogonal planes. MRI is the best diagnostic modality for visualization of the course and insertion of ectopic ureters, and enables precise surgical correction.
\end{abstract}

Keywords: Congenital vaginal ectopic ureter; magnetic resonance imaging; hydrocolpos

\section{CASE REPORT}

A 4-year-old girl presented with a history of intermittent leakage of clear fluid from the genital region. Shortly after birth, she developed a large hydrocolpos causing bilateral ureterohydronephrosis and lower limb edema, and was diagnosed with transverse vaginal septum and a multicystic dysplastic and ectopic right kidney (renal pelvis). On that occasion, drainage and placement of a uterine stoma improved venous and urinary flow. At 10 months, open correction of the vaginal septum and closure of the uterine stoma were performed. After this procedure, the patient developed intermittent dribbling of clear fluid, identical in appearance and odor to urine, from the genital area.

A wide variety of diagnostic tests (including ultrasound, computed tomography [CT], cystoscopy, and vaginoscopy) were performed, but failed to confirm the hypothesis of ectopic ureter draining the dysplastic right kidney. At the age of 3 , the patient was found to have frequent dribbling of urine during diaper changes despite adequate sphincter control. Physical examination revealed passage of fluid droplets through the vaginal introitus, and she was referred for further radiological assessment. A radionuclide kidney scan showed $48 \%$ renal function on the left and complete absence of right kidney function. Dynamic ultrasound examination of the bladder revealed a small amount of fluid in the vagina, which did not coincide with urge to urinate or with recent voiding. Transperineal color Doppler imaging showed discharge of urine into the vagina, suggesting right kidney dysplasia with vaginal drainage of an ectopic
Clin Biomed Res. 2014;34(4):403-405

1 Serviço de Radiologia, Hospital de Clínicas de Porto Alegre (HCPA). Porto Alegre, RS, Brazil.

2 Serviço de Cirurgia Pediatrica, Hospital de Clínicas de Porto Alegre (HCPA). Porto Alegre, RS, Brazil.

3 Serviço de Nefrologia Pediatrica, Universidade Federal de Ciências da Saúde de Porto Alegre (UFCSPA). Porto Alegre, RS, Brazil.

Corresponding author: Juliana Avila Duarte E-mail: juavila2003@yahoo.com.br Serviço de Radiologia Hospital de Clínicas de Porto Alegre Rua Ramiro Barcelos, 2350. 90035-903, Porto Alegre, RS, Brazil. 
ureter. There was compensatory hypertrophy of the left kidney, which had a single collecting system and normal ureter draining into the bladder trigone. The right hemitrigone could not be identified. Magnetic resonance imaging (MRI) was performed in order to confirm the suspected diagnosis. The patient received oral fluid and was asked not to void for 30 minutes before the scan. Neither intravenous contrast nor anesthesia was administered before the study. The mother stayed next to the child during the scan, and the patient was calm and cooperative throughout. T2-weighted turbo spin-echo images were acquired in the axial and coronal planes using a cardiac coil. MRI confirmed the ultrasound findings. The dysplastic right kidney was located between the lower third of the inferior vena cava and the right common iliac vein, with several small cystic areas. The insertion point of the ureter of the dysplastic right kidney was found in the right lateral wall of the upper third of the patient's vagina (figures 1, 2, and 3). Right nephroureterectomy was performed by laparotomy, with removal of the dysplastic kidney and low ligation of the corresponding ureter. The patient recovered uneventfully. At 12-month follow-up, urinary incontinence had improved.

\section{DISCUSSION}

Renal dysplasia with vaginal ectopic ureter is not a frequent event ${ }^{1}$, and the association of this malformation with complete transverse vaginal septum is exceedingly rare ${ }^{2}$. Nevertheless, urinary tract malformations may be associated with genital defects, particularly in females ${ }^{3}$.

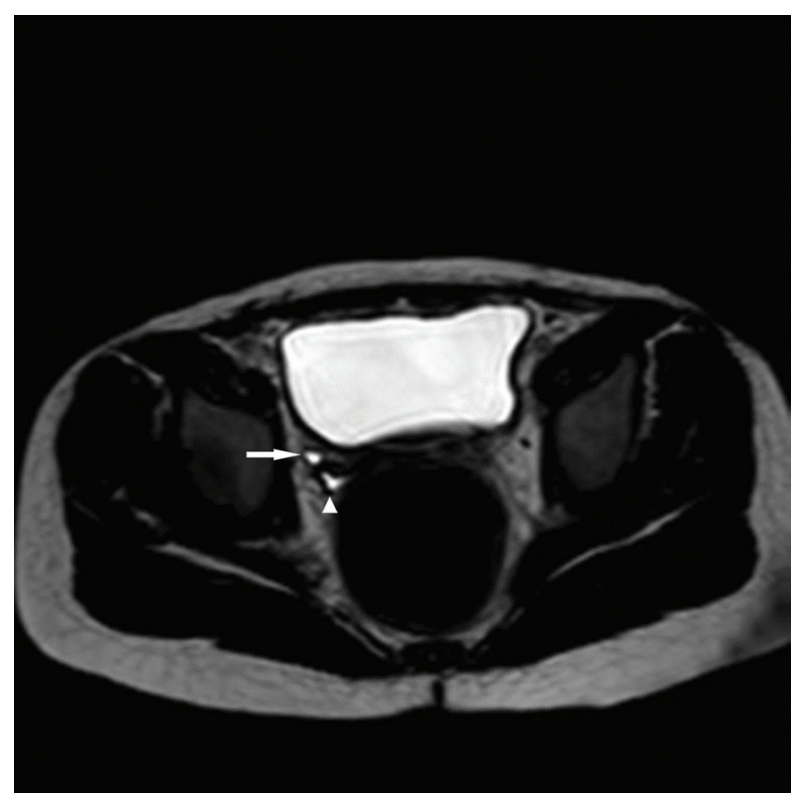

Figure 1: Axial T2-weighted image showing ectopic ureter (arrow) and vaginal fornix (arrowhead)
Single ectopic ureter usually occurs as part of a spectrum of genitourinary tract malformations. Clinical presentation may vary widely depending on which structures are involved and on the extent of involvement. Patients with ureteral ectopia are usually subjected to myriad tests and procedures which fail to confirm the diagnosis - and ineffective pharmacological therapy until a definitive diagnosis is established. Patients with extravesical ectopic ureter usually have urinary leakage despite normal sphincter control, and generally present with a chief complaint of daytime urinary incontinence and nocturnal enuresis, which can have a negative effect on the patient's psychosocial well-being.

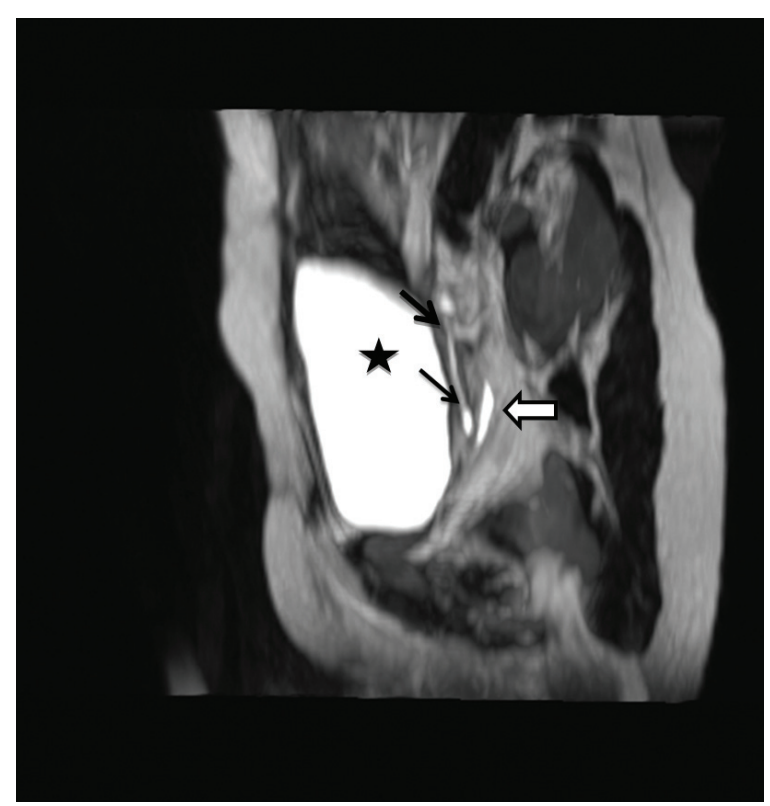

Figure 2: Sagittal T2-weighted image showing ectopic ureter (arrows), anterior wall of the upper third of the vagina (arrowhead), and bladder (star).

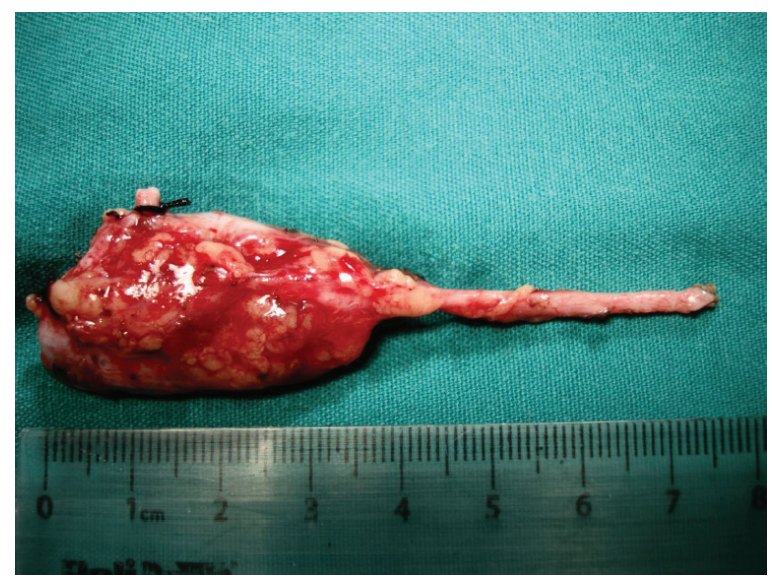

Figure 3: Surgical specimen showing the distal segment of the ectopic ureter draining into the right lateral wall of the upper third of the vagina. 
Ureteral ectopia is difficult to diagnose with conventional imaging methods (plain radiography, ultrasound, and CT). MRI has proved to be an excellent method for assessment of complex genitourinary tract conditions, particularly when other diagnostic modalities have failed or are limited ${ }^{3-6}$. This method is also free of ionizing radiation and provides precise and accurate images of anatomical changes. The high resolution of magnetic resonance (MR) images and the possibility of native image acquisition on all three orthogonal planes, without the need for later reconstruction (as in CT), provides precise knowledge of the location of the diseased structure and anatomical landmarks, thus enabling accurate planning and use of the safest and most appropriate surgical approach for each patient. Nevertheless, the method is not without its disadvantages, which must be considered before it is indicated and include prolonged acquisition time, occasional need for sedation or anesthesia, and use of gadolinium contrast. In the present case, we did not use sedation, anesthesia, gadolinium contrast, or intravenous diuretics. Pretreatment was limited to water intake 1 hour before the scan, which consisted of T1- and T2-weighted gradient echo volumetric images obtained in the axial plane and reconstructed in three orthogonal planes.

The tissue signal of dysplastic kidneys is best demonstrated in T1- and T2-weighted sequences.
Fluid-filled areas of the kidney and ureter appear hyperintense on T2-weighted images, whereas nonfunctioning tissue appears hypointense on T1-weighted sequences ${ }^{5}$. MR has proved superior to conventional imaging modalities for assessment of ureteral ectopia and duplicated systems with a nonfunctioning upper pole ${ }^{4}$. Acquisition of T2-weighted images after fluid intake with or without intravenous furosemide appears to be an effective approach for MR assessment of urinary tract anatomy, as increased diuresis leads to distension (and consequent hyperintensity) of the collecting systems and ureters ${ }^{4,5}$. In a study performed in India, Joshi et al. ${ }^{7}$ reported seven cases of congenital renal dysplasia with single-system ureteral ectopia that were correctly diagnosed by preoperative MR urography, which was found to have $100 \%$ sensitivity and specificity for diagnosis of kidney dysplasia and determination of the course of the ureters.

In conclusion, children presenting with clinical manifestations suggesting ureteral ectopia should undergo MRI investigation, which can confirm diagnosis of this malformation, sparing the child from lengthy diagnostic procedures, and provide essential anatomical information for planning of surgical correction. Proper treatment of this malformation in childhood can minimize its potentially severe psychosocial consequences.

\section{REFERENCES}

1. Moores D, Cohen R, Hayden L. Laparascopic excision of pelvic kidney with single vaginal ectopic ureter. J Pediatr Surg. 1997;32(4):634-5. http://dx.doi.org/10.1016/S00223468(97)90726-0. PMid:9126773

2. Kumar S, Mandal A, Acharya N, Jain V, Kalra J, Singh S. Congenital vesicovaginal fistula with transverse vaginal septum and ectopic ureter opening in proximal vagina: case report and brief review. Int Urogynecol J Pelvic Floor Dysfunct. 2007;18(8):959-61. http://dx.doi. org/10.1007/s00192-006-0258-8. PMid:17143654

3. Wang ZJ, Daldrup-Link H, Coakley FV, Yeh BM. Ectopic ureter associated with uterine didelphys and obstructed hemivagina: preoperative diagnosis by MRI. Pediatr Radiol. 2010;40(3):35860. http://dx.doi.org/10.1007/s00247009-1454-8. PMid:19924410

4. Cerwinka WH, Damien Grattan-Smith J, Kirsch AJ. Magnetic resonance urography in pediatric urology. $J$ Pediatr Urol. 2008;4(1):74-82, quiz 82-3. http://dx.doi.org/10.1016/j. jpurol.2007.08.007. PMid:18631897

5. Sasaki Y, Deguchi E, Tsukada S, Ono $\mathrm{S}$, Iwai $\mathrm{N}$. Usefulness of magnetic resonance imaging for congenital prepubic fistula. J Pediatr Surg. 2010;45(8):1734-6. http://dx.doi. org/10.1016/j.jpedsurg.2010.04.004. PMid:20713231
6. Kibar Y, Avci A, Akay O, Dayanç $M$. Dribbling of urine due to ectopic vaginal insertion of an upper pole ureter diagnosed by magnetic resonance urography. Int Urol Nephrol. 2005;37(4):695-7. http:// dx.doi.org/10.1007/s11255-005-02469. PMid:16362581

7. Joshi M, Parelkar S, Shah H, Sanghvi B, Agrawal A, Mishra P. Role of magnetic resonance urography in the diagnosis of single-system ureteral ectopia with congenital renal dysplasia: a tertiary care center experience in India. $J$ Pediatr Surg. 2009;44(10):1984-7. http://dx.doi. org/10.1016/j.jpedsurg.2009.01.016. PMid:19853759 